\title{
Unnatural amino acids increase activity and specificity of synthetic substrates for human and malarial cathepsin $\mathrm{C}$
}

\author{
Marcin Poreba • Marko Mihelic • Priscilla Krai - Jelena Rajkovic • \\ Artur Krezel · Malgorzata Pawelczak · Michael Klemba • \\ Dusan Turk · Boris Turk · Rafal Latajka $\cdot$ Marcin Drag
}

Received: 3 September 2013/ Accepted: 15 December 2013/Published online: 1 January 2014

(C) The Author(s) 2013. This article is published with open access at Springerlink.com

\begin{abstract}
Mammalian cathepsin $\mathrm{C}$ is primarily responsible for the removal of $\mathrm{N}$-terminal dipeptides and activation of several serine proteases in inflammatory or immune cells, while its malarial parasite ortholog dipeptidyl aminopeptidase 1 plays a crucial role in catabolizing the hemoglobin of its host erythrocyte. In this report, we describe the systematic substrate specificity analysis of three cathepsin C orthologs from Homo sapiens (human), Bos taurus (bovine) and Plasmodium falciparum (malaria parasite). Here, we present a new approach with a tailored fluorogenic substrate library designed and synthesized to probe the S1 and S2 pocket preferences of these enzymes
\end{abstract}

Electronic supplementary material The online version of this article (doi:10.1007/s00726-013-1654-2) contains supplementary material, which is available to authorized users.

M. Poreba $\cdot$ R. Latajka $\cdot$ M. Drag $(\bowtie)$

Division of Bioorganic Chemistry, Faculty of Chemistry,

Wroclaw University of Technology, Wybrzeze Wyspianskiego

27, 50-370 Wrocław, Poland

e-mail: marcin.drag@pwr.wroc.pl

M. Mihelic · J. Rajkovic · D. Turk · B. Turk

Department of Biochemistry and Molecular and Structural

Biology, Jozef Stefan Institute, Ljubljana, Slovenia

P. Krai · M. Klemba

Department of Biochemistry, Virginia Tech, Blacksburg, VA 24061, USA

\section{A. Krezel}

Laboratory of Chemical Biology, Faculty of Biotechnology, University of Wrocław, ul. Joliot-Curie 14a, 50-383 Wrocław, Poland

M. Pawelczak

Faculty of Chemistry, University of Opole, ul. Oleska 48, 45-052 Opole, Poland with both natural and a broad range of unnatural amino acids. Our approach identified very efficiently hydrolyzed substrates containing unnatural amino acids, which resulted in the design of significantly better substrates than those previously known. Additionally, in this study significant differences in terms of the structures of optimal substrates for human and malarial orthologs are important from the therapeutic point of view. These data can be also used for the design of specific inhibitors or activity-based probes.

Keywords Cysteine protease - Non-proteinogenic . Unnatural amino acid · Substrate library $\cdot$ Fluorogenic substrate

\section{Introduction}

The specificity ratio between enzyme orthologs or homologs is one of the most important factors in terms of drug design or specific enzyme activity monitoring using chemical probes. For example, there are several families of proteases, such as caspases, cathepsins or aminopeptidases, which are able to cleave the same peptide sequences, which significantly complicate their targeting with specific chemical tools, such as substrates or activity-based probes. This goal is even more complicated when trying to design specific molecules for monitoring enzyme orthologs, for example, in the case of parasite infection in humans. An excellent example of this difficulty is human cathepsin $\mathrm{C}$ and its malarial ortholog dipeptidyl aminopeptidase 1 (DPAP1).

Cathepsin C (DPPI, EC 3.4.14.1, dipeptidyl peptidase I) is a lysosomal cysteine protease expressed in the majority of mammalian tissues (Tallan et al. 1952). It is considered a major coordinator for the activation of several serine 
proteases (cathepsin G, cytotoxic lymphocyte derived granzymes $\mathrm{A}$ and $\mathrm{B}$, neutrophil elastase) in immune/ inflammatory cells (Turk et al. 2001; Pham and Ley 1999; McGuire et al. 1993). Defects in cathepsin C expression lead to several disorders, including Haim-Munk and Papillon-Lefevre syndromes (Hart et al. 1999, 2000). Other studies report the involvement of cathepsin $\mathrm{C}$ in cytotoxic lymphocyte-mediated apoptosis, angiogenesis or host immune defense (Gocheva and Joyce 2007; Adkison et al. 2002). Structurally, human cathepsin C is a homotetramer ( $\sim 200 \mathrm{kDa})$ comprising four identical catalytically active subunits (Dahl et al. 2001; Turk et al. 2001). Each subunit contains a light chain, a heavy chain and an exclusion domain. Mechanistically, cathepsin $\mathrm{C}$ is a classic exopeptidase, which trims dipeptides from the N-terminus of peptide substrates.

The malarial ortholog of mammalian cathepsin $\mathrm{C}$, dipeptidyl aminopeptidase 1 (DPAP1), is a cysteine protease, which most efficiently hydrolyzes amide bonds at acidic pH (Wang et al. 2011). DPAP1 is located in food vacuoles and plays the role of an intermediate protease between the endopeptidase and aminopeptidase activities (Kudo et al. 2012). Specifically, according to current hypotheses, DPAP1 hydrolyzes the peptide sequences generated by three classes of endopeptidases: aspartic proteases (plasmepsins), cysteine proteases (falcipains) and metalloproteases (falcilysin). This leads to short peptide fragments, which are further hydrolyzed into single amino acids in the vacuole by the metal-dependent M1-family aminopeptidase, PfA-M1 (Ragheb et al. 2011). These amino acids are either used by parasites for protein synthesis or are released by the parasite into the surrounding media (Krugliak et al. 2002). In contrast to humans, parasite genomes contain another two DPAP1-related enzymes, DPAP2 and DPAP3. DPAP3 inhibition leads to the blockage of parasite egress (Arastu-Kapur et al. 2008). A recent work by Tanaka et al. demonstrates that DPAP2 is active in gametocyte. It also showed that the DPAP2 KO in $P$. falciparum or $P$. berghei has no effect on parasite development, thus indicating that DPAP2 is not essential (Tanaka et al. 2013).

The implication of cathepsin C and DPAP1 in pathological disorders makes both enzymes very interesting medicinal targets. To date, human cathepsin $\mathrm{C}$ has especially been investigated, with several chemical approaches leading to potent substrates, inhibitors and activity-based probes (Yuan et al. 2006; Guay et al. 2010). DPAP1 has been less extensively investigated. The substrate specificity of both enzymes, interrogated with a combinatorial library of fluorogenic dipeptides containing natural amino acids, revealed some differences in the recognition of the $S 1$ and S2 subsites, but few significant differences between the enzymes have been found (Wang et al. 2011).
In this report, we have designed and synthesized a fluorogenic dipeptide substrate library containing all natural amino acids (except cysteine, which is prone to oxidation) and several structurally different unnatural amino acids. We hypothesized that the application of such a broad range of different amino acid structures would help to identify more significant differences in the optimal substrates recognized by human and malarial cathepsin $\mathrm{C}$ and to design more active substrates in terms of kinetic parameters. To obtain better insight into cathepsin $\mathrm{C}$ orthologs, in addition to human and malarial cathepsin $\mathrm{C}$, we also analyzed the substrate specificity of the bovine (Bos Taurus) ortholog.

The approach presented here can be generally used for the substrate specificity screening of other diaminopeptidases. When the structure of an enzyme is not available, information from the library can be used to predict the size of and preferences for the $\mathrm{S} 1$ and $\mathrm{S} 2$ pockets and can be further applied to the design of specific substrates or inhibitors. We also demonstrate that this approach allows the differentiation of enzyme orthologs from different species, yielding information about evolutionary changes.

\section{Materials and methods}

\section{General}

Fmoc-Rink-amide AM polystyrene resin (mesh 100-200, $0.64 \mathrm{mmol} / \mathrm{g}$ ), piperidine, $O$-(benzotriazol-1-yl)- $N, N, N^{\prime}, N^{\prime}$ tetramethyluronium hexafluorophosphate (HBTU) and trifluoroacetic acid (TFA) were purchased from Iris Biotech GmbH. Anhydrous N,N-dimethylformamide (DMF) was purchased from J. T. Baker. Dichloromethane (DCM), methanol $(\mathrm{MeOH})$ and diethyl ether $\left(\mathrm{Et}_{2} \mathrm{O}\right)$ were purchased from POCH S.A. (Poland). Fmoc-protected amino acids were purchased from Sigma Aldrich, Iris Biotech $\mathrm{GmbH}$, Fluka and Novabiochem. $O$-(7-azabenzotriazol-1-yl)$\mathrm{N}, \mathrm{N}, \mathrm{N}^{\prime} \mathrm{N}^{\prime}$-tetramethyluronium hexafluorophosphonate (HATU) was purchased from Novabiochem. 2,4,6-trimethylpyridine (collidine), N,N-diisopropylethylamine (DIPEA), triisopropylsilane (TIPS), DEAE-Sepharose, Sephadex G-200, 2-mercaptoethanol, diisopropyl phosphorofluoridate (DFP), bovine serum albumin and EDTA$\mathrm{Na}_{2}$ were purchased from Sigma Aldrich (USA). Molecular weight calibration markers for gel filtration and protein markers for SDS-PAGE were purchased from Bio-Rad (USA). Gly-Phe-pNA was a gift from Dr. Maciej Makowski, Department of Chemistry, University of Opole (Opole, Poland). All chemicals and solvents were used without further purification. 7-Fmoc-aminocoumarin-4acetic acid was synthesized in our laboratory according to the procedure described previously (Maly et al. 2002). 
Human, bovine and malarial cathepsin $\mathrm{C}$ expression and purification

Human cathepsin $\mathrm{C}$ was expressed according to the procedure described elsewhere (Dahl et al. 2001). Cathepsin C was activated by cathepsin $\mathrm{L}$ (molar ratio 20:1, respectively) in the following buffer: $20 \mathrm{mM}$ citric acid, $150 \mathrm{mM} \mathrm{NaCl}$, $1 \mathrm{mM}$ EDTA and $5 \mathrm{mM}$ DTT, $\mathrm{pH}$ 4.5. Cathepsin $\mathrm{C}$ was activated for $4 \mathrm{~h}$. The protein mixture was loaded onto a preparative HiLoad Superdex 200 size-exclusion column using fast protein liquid chromatography (AKTA Purifier, $1.6 \mathrm{~cm} \times 60 \mathrm{~cm}$, GE Healthcare, Sweden) equilibrated with $50 \mathrm{mM}$ sodium acetate, $1 \mathrm{mM}$ EDTA and $300 \mathrm{mM} \mathrm{NaCl}$, $\mathrm{pH} 5.5$, at a flow rate of $1.2 \mathrm{~mL} / \mathrm{min}$ and $5^{\circ} \mathrm{C}$. The protein was analyzed by SDS-PAGE, and fractions containing cathepsin $\mathrm{C}$ were collected and concentrated to $5 \mu \mathrm{M}$. Active site titration was carried out as described in Online Resource 2. The enzyme was $65 \%$ active. Cathepsin $\mathrm{C}$ concentrations and $k_{\text {cat }}$ values are given per enzyme complex.

Bovine cathepsin C (DPP I; EC 3.4.14.1) was purified from bovine spleen after acid extraction, heat treatment, ammonium sulfate fractionation, gel filtration chromatography and ion-exchange chromatography. Purification was carried out according to the method developed by McDonald et al. and supplemented with an ion-exchange chromatography step on a column of DEAE-Sepharose (Ken McDonald et al. 1972). The purified enzyme showed a native molecular mass of $\sim 200 \mathrm{kDa}$ by Sephadex G-200 column chromatography. Enzyme concentration was calculated based on total protein.

Purified recombinant $P$. falciparum dipeptidyl aminopeptidase 1 (rDPAP1) was generated as described in Wang et al. (2011). Enzyme concentration was calculated based on total protein. DPAP1 concentrations, and $\mathrm{k}_{\mathrm{cat}}$ values are given per enzyme complex.

Synthesis of the substrate library

$\mathrm{NH} 2-\mathrm{ACC}$ resin was prepared by the reaction of AmideRink resin with the 7-Fmoc-aminocoumarin-4-acetic acid. The resin was first swollen in anhydrous dichloromethane for an hour and washed with DMF, and the Fmoc-protecting group was removed by $20 \%$ piperidine/80 \% DMF $(25,5$, $5 \mathrm{~min})$. This prepared resin was washed three times with DMF. Next, deprotected resin was dissolved in DMF, and Fmoc-ACC-OH (2.0 eq.), HBTU (2.0 eq.) and DIPEA (2.0 eq.) were added. The mixture was agitated for $24 \mathrm{~h}$, filtered and washed ( 3 times with DMF). The resin was then redissolved in DMF, and the second coupling was performed with Fmoc-ACC-OH (1.0 eq.), HBTU (1.0 eq.) and DIPEA (1.0 eq.). The mixture was agitated for the next $24 \mathrm{~h}$, filtered and washed ( 3 times with DMF). The substitution level after the second coupling was $>98 \%$. The Fmoc-ACC resin was deprotected with $20 \%$ piperidine/80 \% DMF (25, 5, $5 \mathrm{~min})$, filtered, washed ( 3 times with DMF, 3 times with DCM and 3 times with $\mathrm{MeOH}$ ) and dried over $\mathrm{P}_{2} \mathrm{O}_{5}$. The NH2-ACC resin was used to construct both the $\mathrm{P} 1$ and the $\mathrm{P} 2$ fluorogenic substrate libraries.

\section{P1 library}

Dried $\mathrm{NH}_{2}$-ACC resin was split into 36 portions (100 mg each) and placed into the wells of a 96-well semiautomatic FlexChem synthesizer. The resin was then swollen in anhydrous DCM for an hour. Next, the $\mathrm{NH}_{2}-\mathrm{ACC}$ resin was filtered, washed (3 times with DMF) and solvated in DMF. An individual Fmoc-amino acid-OH (2.5 eq.), HATU (2.5 eq.) and collidine (2.5 eq.) were sequentially added to the wells, and the reaction was agitated for $24 \mathrm{~h}$, filtered and washed (3 times with DMF). The second coupling was as follows: individual Fmoc-amino acid-OH (1.0 eq.), HATU (1.0 eq.) and collidine (1.0 eq.). The mixture was shaken for the next $24 \mathrm{~h}$, filtered and washed (3 times with DMF). The Fmoc-protecting group was removed by $20 \%$ piperidine $/ 80 \% \operatorname{DMF}(25,5,5 \mathrm{~min})$, and the resin was washed three times with DMF. In the P2 position, L-methionine was fixed. The resin was swollen in anhydrous DMF, and Fmoc-L-Met-OH (2.5 eq.), HBTU (2.5 eq.) and DIPEA (2.5 eq.) were added. The mixture was shaken for $3 \mathrm{~h}$, filtered and washed (3 times with DMF). Next, the Fmoc-protecting group was removed as previously described. The resin was filtered, washed three times with DMF, three times with DCM, three times with $\mathrm{MeOH}$ and dried over $\mathrm{P}_{2} \mathrm{O}_{5}$. Finally, the dry $\mathrm{NH}_{2}$-L-Met-XACC resin was obtained. The cold mixture of TFA (95\%), water $(2.5 \%)$ and TIPS $(2.5 \%)$ was used to remove the P1 library from the resin (agitating for $2 \mathrm{~h}$ ). Each single substrate was precipitated in cold ether and centrifuged. After decantation, each substrate was dissolved in DMSO and purified by HPLC on a Waters M600 solvent delivery module with a Waters M2489 detector system using a semi-preparative Waters Spherisorb S10ODS2 column. The solvent composition was as follows: phase A (water/ $0.1 \%$ TFA) and phase B (acetonitrile/water $80 \% / 20 \%$ (v/ v) with $0.1 \%$ of TFA). The purity of each substrate was confirmed by analytical HPLC using a Waters Spherisorb S5ODS2 column. All compounds were at least $95 \%$ pure. Each of the 36 dipeptidyl fluorogenic substrates was lyophilized, weighed and dissolved in DMSO to a final concentration of $20 \mathrm{mM}$. Finally, the molecular weight of each substrate was confirmed by ESI-MS analysis.

\section{P2 library}

Dry $\mathrm{NH}_{2}$-ACC resin was split into 57 portions $(100 \mathrm{mg}$ each) and placed into the wells of a 96-well 
semiautomatic FlexChem synthesizer. Then, the resin was swollen in anhydrous DCM for an hour. Next, the $\mathrm{NH}_{2^{-}}$ ACC resin was filtered, washed (3 times with DMF) and solvated with DMF. The P1 position was fixed with Lhomophenylalanine as follows: Fmoc-L-hPhe-OH (2.5 eq.), HATU (2.5 eq.) and collidine (2.5 eq.) were added to each well, and the mixtures were shaken for $24 \mathrm{~h}$, filtered, washed (3 times with DMF) and redissolved in DMF. In the second coupling, Fmoc-L-hPhe-OH (1.0 eq.), HATU (1.0 eq.) and collidine (1.0 eq.) were added to each well, and the mixtures were agitated for the next $24 \mathrm{~h}$, filtered and washed as previously described. The substitution level after the second coupling was $>95 \%$ (HPLC analysis). Next, Fmoc-L-hPhe-ACC resin was deprotected with $20 \%$ piperidine/ $80 \% \operatorname{DMF}(25,5$, $5 \mathrm{~min}$ ), and the resin was washed three times with DMF. The P2 position was substituted with individual Fmocamino acid-OH as follows: Fmoc-amino acid-OH (2.5 eq.), HBTU (2.5 eq.) and DIPEA (2.5 eq.) were added to individual wells containing solvated (DMF) $\mathrm{NH}_{2}$-L-hPhe-ACC resin. The mixtures were agitated for $3 \mathrm{~h}$, filtered and washed ( 3 times with DMF). The Fmocprotecting group was removed with $20 \%$ piperidine $/ 80 \%$ $\operatorname{DMF}(25,5,5 \mathrm{~min})$, and the resin was washed three times with DMF, three times with DCM and three times with $\mathrm{MeOH}$ and dried over $\mathrm{P}_{2} \mathrm{O}_{5}$. The cold mixture of TFA $(95 \%)$, water $(2.5 \%)$ and TIPS $(2.5 \%)$ was used to remove the P2 library from the resin. Each single substrate was precipitated in cold ether and centrifuged. After the decantation and lyophilization, each substrate was dissolved in DMSO to a final concentration of $20 \mathrm{mM}$. There was no need to further purify the substrates because the substitution level after coupling L-hPhe was at least $95 \%$, and the P2 coupling reaction occurs with a $100 \%$ yield. The purity of each single substrate was confirmed by analytical HPLC using a Waters Spherisorb S5ODS2 column. The solvent composition was as follows: phase A (water $/ 0.1 \%$ TFA) and phase B (acetonitrile/water $80 \% /$ $20 \%$ (v/v) with $0.1 \%$ of TFA).

Assay of the substrate library

The P1 and P2 fluorogenic substrate libraries were screened against cathepsin $\mathrm{C}$ from two mammals (Homo sapiens and Bos taurus) and one protozoan parasite (Plasmodium falciparum). The P1 library $\left(\mathrm{NH}_{2}\right.$-L-Met-XACC) consists of 36 individual compounds, and the $\mathrm{P} 2$ library $\left(\mathrm{NH}_{2}-\mathrm{X}\right.$-L-hPhe-ACC) consists of 57 individual compounds. Human cathepsin $\mathrm{C}$ was assayed in the following buffer: $100 \mathrm{mM}$ sodium acetate, $100 \mathrm{mM} \mathrm{NaCl}$, $1 \mathrm{mM}$ EDTA, and $5 \mathrm{mM}$ DTT, pH 5.5. Bovine spleen cathepsin $\mathrm{C}$ was activated in $170 \mathrm{mM} \mathrm{NaCl}$ solution containing $1 \mathrm{mM}$ EDTA and $1 \mathrm{mM}$ DTT at $37{ }^{\circ} \mathrm{C}$ for
$0.5 \mathrm{~h}$. The enzyme was then assayed in $0.1 \mathrm{M}$ acetate buffer containing $30 \mathrm{mM} \mathrm{NaCl}, 1 \mathrm{mM}$ EDTA and $1 \mathrm{mM}$ DTT, pH 5.0. Buffers for the screening of mammalian cathepsin $\mathrm{C}$ orthologs were prepared at $23{ }^{\circ} \mathrm{C}$, and assays were conducted at $37^{\circ} \mathrm{C}$.

Assays of DPAP1 were conducted in $50 \mathrm{mM}$ Na-MES, pH 6, $30 \mathrm{mM} \mathrm{NaCl}, 1 \mathrm{mM}$ EDTA, $2 \mathrm{mM}$ DTT and $0.1 \%$ Triton X-100 at $25{ }^{\circ} \mathrm{C}$. Each library compound was assayed at a concentration of $1 \mu \mathrm{M}$. The library was screened using a DPAP1 concentration of $2 \mathrm{nM}$. To obtain reliable rates with the most efficiently cleaved substrates, the enzyme concentration needed to be reduced to $0.5 \mathrm{nM}$. Rates from these reactions were multiplied by a factor of four to enable comparison with rates obtained with $2 \mathrm{nM}$ enzyme. The background rate was determined in an enzyme blank containing $1.0 \mu \mathrm{M}$ Val-Arg-ACC (Wang et al. 2011) and was subtracted from all enzymatic rates.

Before being added to the substrate, cathepsins were preincubated at $37^{\circ} \mathrm{C}$ for $30 \mathrm{~min}$. The final library concentration was $1 \mu \mathrm{M}$. Enzyme concentrations were between 1 and $5 \mathrm{nM}$.

The hydrolysis of ACC substrates was monitored continuously with an excitation wavelength of $355 \mathrm{~nm}$ and an emission wavelength of $460 \mathrm{~nm}$ using a Spectra MAX Gemini EM fluorimeter (Molecular Devices). The total time of each assay was between 5 and $15 \mathrm{~min}$. From each single experiment, the linear portion of the progress curve was used to calculate the final substrate rate of hydrolysis and reported as the relative fluorescence unit per second (RFU/s). Each experiment was repeated at least three times, and the standard error of measurements was calculated. The average value for each substrate was compared with the best-cleaved substrate of a given library. All data were presented on a two-dimensional graph, where the $x$ axis represents individual fluorogenic substrates and the $y$ axis represents the production of relative fluorescence units set to $100 \%$ for Met-Arg-ACC in P1 and Arg-hPhe in P2 library.

Determination of kinetic parameters

$\left(k_{\mathrm{cat}}, K_{\mathrm{m}}\right.$, and $\left.k_{\mathrm{cat}} / K_{\mathrm{m}}\right)$ for individual substrates

Selected substrates were analyzed against human cathepsin $\mathrm{C}$ with the above assay buffers. Before being added to the substrate, all enzymes were preincubated at $37{ }^{\circ} \mathrm{C}$ for $30 \mathrm{~min}$. The ACC final concentration was calculated by a total digestion assay for human cathepsin C. In each measurement, ten independent substrates with known concentrations were chosen, and the average value was calculated. To measure the $K_{\mathrm{m}}$ value, eight different concentrations of the given substrates and constant enzyme concentrations were used. The reaction volume was $100 \mu \mathrm{L}$, and the enzyme concentration was $1.0 \mathrm{nM}$ 


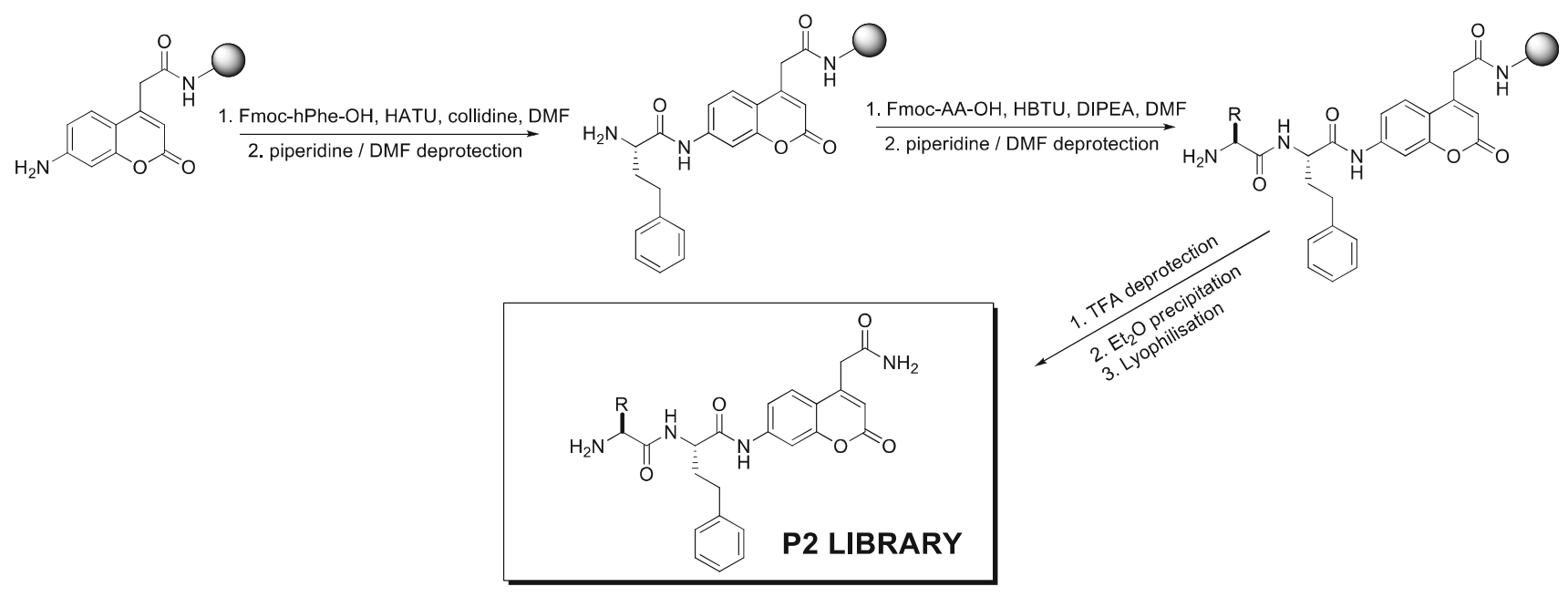

Fig. 1 Synthesis and structure of P2 library

for human cathepsin C. All experimental conditions were as above. The hydrolysis of ACC substrates was monitored as in the previous section. The total time of each assay was between 10 and $30 \mathrm{~min}$. All experiments were repeated at least three times, and the average value with standard deviation was calculated. The concentration of DMSO in each experiment was $<1 \%(\mathrm{v} / \mathrm{v})$. For DPAP1, kinetic analyses were conducted in triplicate as previously described using 2 nM DPAP1 (Wang et al. 2011). We have also calculated the kinetic parameters for human cathepsin $\mathrm{C}$ using optimal cathepsin $\mathrm{C}\left(\mathrm{NH}_{2}-\mathrm{Abu}-\right.$ $\mathrm{Nle}(\mathrm{OBzl})-\mathrm{ACC}$ ) and DPAP1 (Pip-Lys-ACC) substrates at different $\mathrm{pH}$, DTT and $\mathrm{NaCl}$ concentrations as well as in optimal condition for each enzyme buffer. They are attached in Online Resource.

\section{Results}

Design of the P1 and P2 dipeptide libraries

To use unnatural amino acids in our approach, we have not used the classic positional scanning substrate combinatorial library (PS-SCL) methodology due to its use of only natural amino acids. In our approach, we have synthesized the $\mathrm{P} 2$ library of substrates by fixing in the $\mathrm{P}_{1}$ position $\mathrm{L}-\mathrm{hPhe}$ (L-homo-phenylalanine), an amino acid described in previous reports as one of those most preferred by cathepsin $\mathrm{C}$ (Li et al. 2009). L-hPhe was coupled (double coupling) with more than $95 \%$ yield to ACC fluorophore (7-amino-4carbamoylmethylcoumarin) linked to Rink-amide resin, according to previously described methodology (Fig. 1) (Maly et al. 2002).
We used the ACC fluorophore as the leaving group because of its convenience in solid phase synthesis (Maly et al. 2002). Next, $\mathrm{NH}_{2}-\mathrm{L}-\mathrm{hPhe}-\mathrm{ACC}$ was split, and the parallel coupling of Fmoc-protected amino acids using a semiautomatic FlexChem synthesizer was performed, yielding individual substrates after cleavage.

In this library, which consisted of 57 individual substrates, we used all natural amino acids (except L-cysteine due to its susceptibility to oxidation), several L-amino acid enantiomers (D-amino acids) and a broad range of unnatural amino acids, of which the structures were chosen to cover a spectrum of the possible interactions in the $\mathrm{S} 2$ pocket of human cathepsin $\mathrm{C}$ (full structures are in Online Resource 1). The purity of the substrates was confirmed using analytical HPLC. Finally, we screened the whole library at a substrate concentration of $1 \mu \mathrm{M}$, which was sufficiently below the lowest $K_{\mathrm{m}}$ of all tested substrates to ensure that velocity data are proportional to $k_{\text {cat }} / K_{\mathrm{m}}$.

Having determined the $\mathrm{P} 2$ preference of cathepsin $\mathrm{C}$, we next designed a library to screen the substrate specificity in the $\mathrm{S} 1$ pocket of this enzyme. In the first step, we obtained the ACC fluorophore linked to Rink-amide resin. Next, this resin was split, and double coupling of the first amino acid was performed using previously described methodology with a semiautomatic FlexChem synthesizer. In the P2 position, we fixed the optimal natural amino acid determined in the previous step, L-Met (Fig. 2). To build this library consisting of 36 individual substrates, we applied all natural amino acids (except L-cysteine) and several unnatural amino acids. However, based on previous reports, we applied mostly unnatural amino acids with bulky and hydrophobic side chains [Bpa, Bip, Nle( $O$-Bzl), hPhe, Glu(Bzl)] (full structures are in Online Resource 1) 


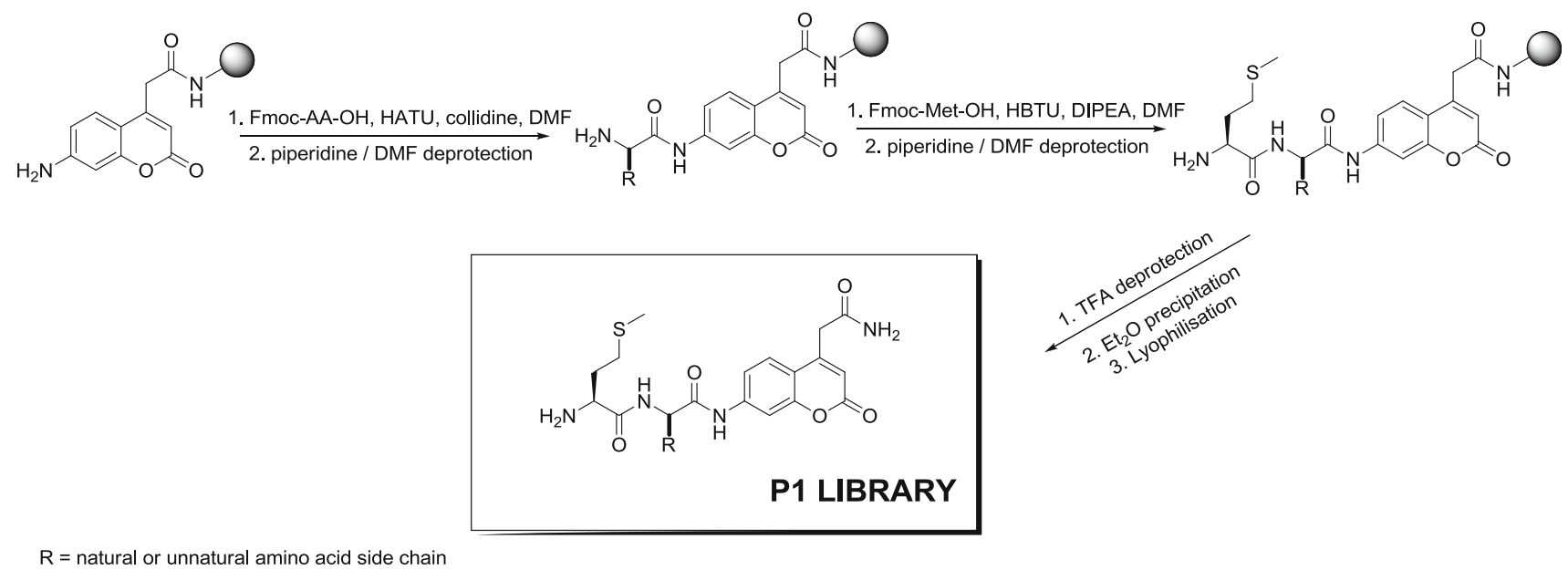

Fig. 2 Synthesis and structure of the P1 library

(Li et al. 2009). Finally, all the substrates were cleaved from the resin and purified using preparative HPLC and analyzed using analytical HPLC.

Exactly as described above, we determined the preliminary conditions $\left(K_{\mathrm{m}}\right)$ of all cleaved substrates and performed the parallel screening of the library at a final substrate concentration of $1.0 \mu \mathrm{M}$.

Substrate specificity analysis of human and bovine cathepsin C and DPAP1

Bovine cathepsin $\mathrm{C}$ is often used to mimic human cathepsin C. We applied our library to compare both enzymes directly in terms of substrate specificity. The analysis of the $\mathrm{S} 1$ pocket preferences of mammalian cathepsin $\mathrm{C}$ demonstrates that these enzymes recognize exactly the same residues at almost the same level (Fig. 3a, b). The most preferred amino acids can be assigned to one of the following groups: hydrophobic [Nle( $O$-Bzl), Bpa, Bip, Tyr(Bzl), Glu(Bzl), hPhe], basic (Arg, Lys) or aliphatic (Nva, Met, Leu). This finding demonstrates that the $\mathrm{S} 1$ pocket size is much larger than the natural amino acids and can very easily accommodate more bulky residues, clearly confirming a conserved level of structure organization of these enzymes.

This observation is also in agreement with data published by $\mathrm{Li}$ et al. (2009), which demonstrated that some unnatural amino acids (L-hPhe or L-Bpa) bind much better than L-Phe (Fig. 3). Among natural amino acids, the ones best tolerated by human and bovine cathepsin $\mathrm{C}$ were Arg, Lys, Gln and Met. These data are in quite good agreement with previously published data by Wang et al. (2011), who applied a combinatorial library approach. However, it needs to be underlined that the best natural amino acid (Arg) was recognized only at $\sim 20 \%$ compared to the best unnatural derivative from our library (L-Nle $(O$-Bzl $)$ ).
Malarial DPAP1 substrate specificity in the $\mathrm{S} 1$ pocket is much more restricted compared to the mammalian orthologs tested here. DPAP1 preferentially recognizes and hydrolyzes such amino acids like $\mathrm{Nle}(O-\mathrm{Bzl})$, Lys, Glu(Bzl), Arg, Met, Gln, Thr, hPhe and Nva (Fig. 3c). These data are in quite good agreement with the substrate specificity of mammalian orthologs. The most striking difference can be observed in the case of large and bulky unnatural amino acids (Bip, Bpa, Cha). DPAP1 very minimally hydrolyzes these derivatives, which clearly demonstrates the difference in $\mathrm{S} 1$ pocket preferences between mammalian and parasite orthologs.

The analysis of the S2 pocket of both mammalian orthologs demonstrates, similar to the case of the S1 pocket, a very high level of agreement in the activity and tolerance of amino acids (Fig. 4a, b).

The amino acids best recognized by both enzymes were Abu, Hse, Met, Nva, Nle and Ala. All these amino acids are rather small and have aliphatic chains. Bulky and hydrophobic side chains were practically unrecognized by either enzyme in the S2 pocket. No activity of human cathepsin C toward amino acids with basic side chains (Arg, Lys, Orn, Dap and Dab) was observed, in agreement with previously published data (Wang et al. 2011). Additionally, none of the D-amino acids was recognized and hydrolyzed by mammalian orthologs, which indicates the high stereospecificity of both enzymes around the S2 binding pocket (structures in Online Resource 1). Although we found slightly different overall ratios from the report by Wang et al. (2011) with regard to natural amino acids, our values are in quite good agreement in terms of overall amino acid preferences. The differences observed can result from various screening conditions or the composition of the library.

The substrate specificity analysis of malarial DPAP1 demonstrates a very striking difference from mammalian 


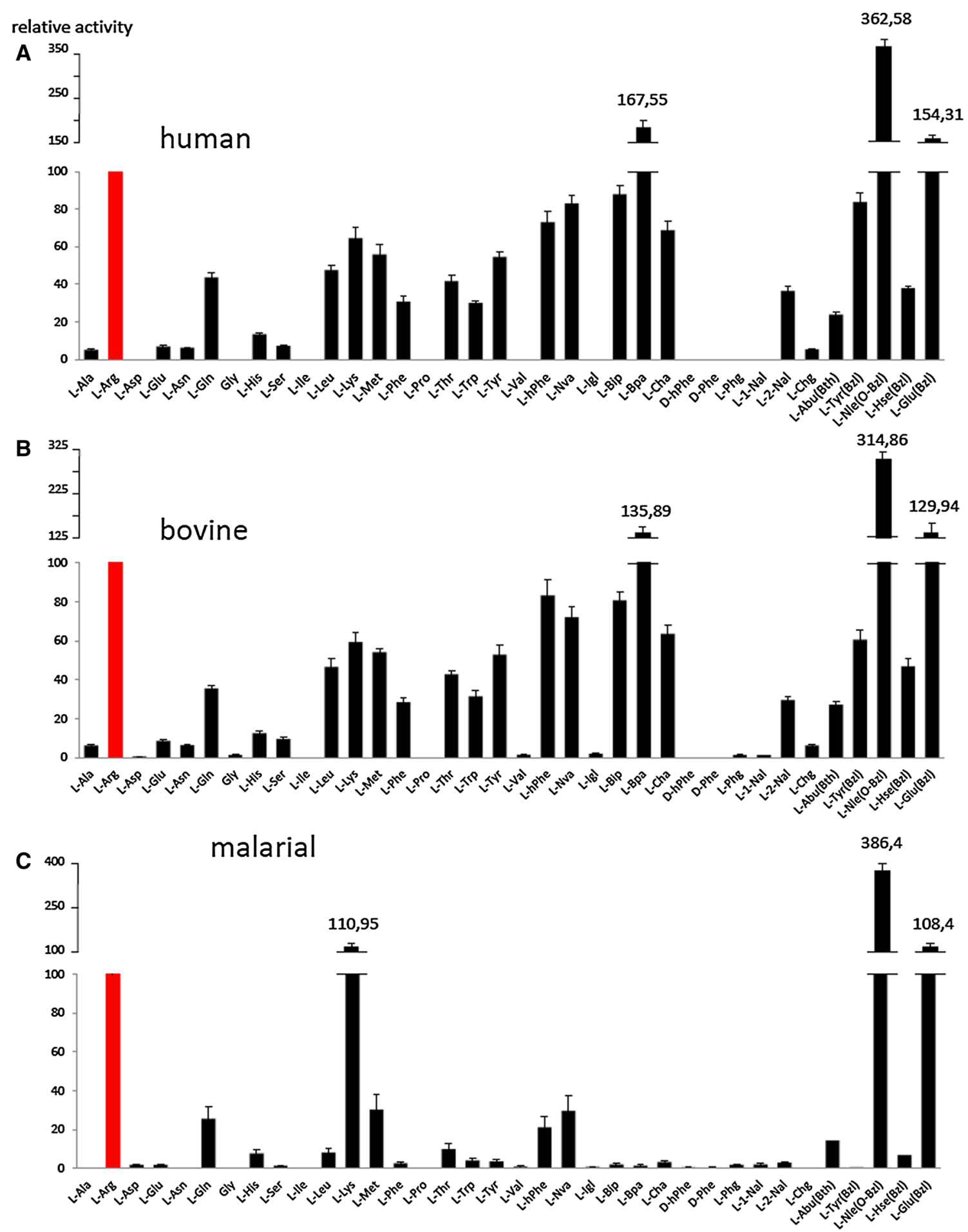

Fig. 3 Substrate specificity of human and bovine cathepsin $\mathrm{C}$ and malarial DPAP1 in the S1 pocket (substrate concentration $1 \mu \mathrm{M}$, human and bovine cathepsin $\mathrm{C}-3 \mathrm{nM}$, rDPAP1-2 nM). Proteinogenic and unnatural amino acid abbreviations are shown on the $x$-axis.

orthologs. L-Pip, a six-membered cyclic unnatural homo$\log$ of proline with one extra methylene residue, was the best tolerated in the $\mathrm{S} 2$ pocket. Substrates with this residue were recognized at least twice as well by DPAP1 than the
The $y$-axis represents the average relative activity as a percentage of the L-Arg substrate activity. All the results were normalized to L-Arg. All structures and information about fluorogenic substrates are in Online Resource 1

second best-recognized amino acid, Abu, and almost twoand-a-half times better than the best-recognized natural amino acid, Val. Data obtained for natural amino acids are also in quite good agreement with these previously 


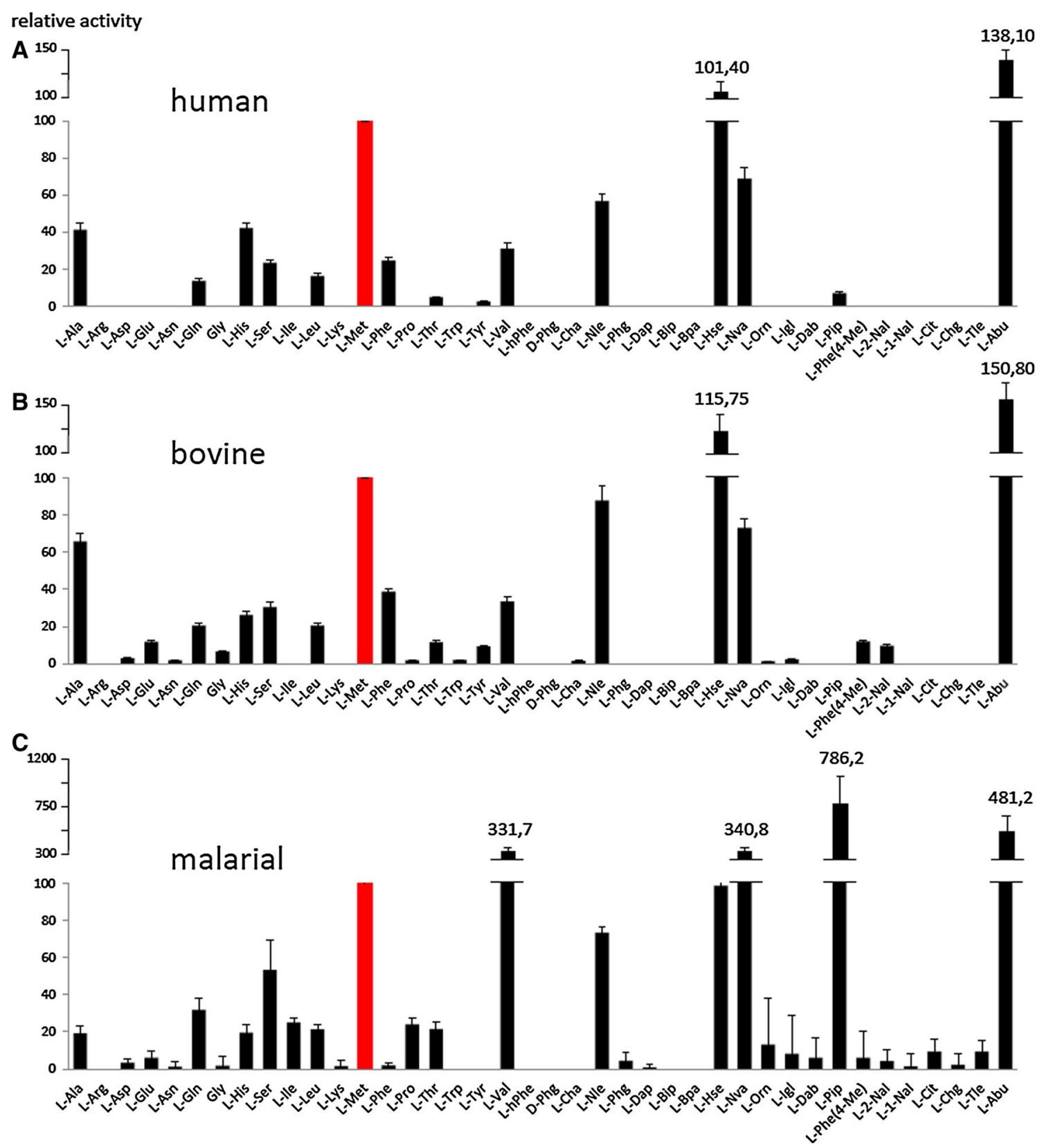

Fig. 4 Substrate specificity of human and bovine cathepsin $\mathrm{C}$ and malarial DPAP1 in the S2 pocket (substrate concentration $1 \mu \mathrm{M}$, human and bovine cathepsin C-3 nM, DPAP1-2 nM). Proteinogenic and unnatural amino acid abbreviations are shown on the $x$-axis. The $y$-axis represents the average relative activity as a percentage of

published (Wang et al. 2011). Another interesting finding was that this amino acid was barely recognized by either mammalian ortholog, which gives hope in the design of specific substrates or inhibitors. A great example here are the data obtained with the inhibitor specificity profile reported by Arastu-Kapur et al., where a library of dipeptide vinyl-sulfone inhibitors containing natural and nonnatural amino acids in the $\mathrm{P} 2$ position was screened against the L-Met substrate activity. D-amino acids, which were not recognized by any of the tested enzymes, are not shown here. All the results were normalized to methionine. All structures and information about fluorogenic substrates are in Online Resource 1

DPAP1, DPAP3 and the falcipains. In this study, inhibitor with Pro in P2 position was found to be specific for DPAP1 (Arastu-Kapur et al. 2008). In addition to L-Pip, other natural and unnatural amino acids were recognized at a decent rate by DPAP1. These amino acids were Abu, Val, Met, Nva, Hse and Ala. Similarly, as in the case of mammalian orthologs, D-amino acids were not recognized and hydrolyzed by DPAP1. 
Table 1 Kinetic parameters $\left(K_{\mathrm{m}}, k_{\mathrm{cat}}, k_{\mathrm{cat}} / K_{\mathrm{m}}\right)$ of selected substrates for human cathepsin $\mathrm{C}$ from the P1 library $\left(\mathrm{NH}_{2}\right.$-L-Met-X-ACC). Each measurement was repeated at least three times

\begin{tabular}{|c|c|c|c|c|}
\hline \multicolumn{2}{|l|}{$\mathrm{NH}_{2}$-Met-X-ACC } & \multicolumn{3}{|c|}{ Human cathepsin $\mathrm{C}$} \\
\hline $\mathrm{X}$ : code/name & $\mathrm{X}$ : structure & $K_{\mathrm{m}} \mu \mathrm{M}$ & $k_{\text {cat }} \mathrm{s}^{-1}$ & $k_{\text {cat }} / K_{\mathrm{m}} \times 10^{5} \mathrm{~s}^{-1} \mathrm{M}^{-1}$ \\
\hline Arg (arginine) & & $7.49 \pm 0.61$ & $12.80 \pm 0.08$ & $17.2 \pm 0.15$ \\
\hline hPhe (homophenylalanine) & & $3.46 \pm 0.09$ & $5.63 \pm 0.27$ & $16.1 \pm 0.15$ \\
\hline Bip (biphenylalanine) & & $4.11 \pm 0.13$ & $6.28 \pm 0.61$ & $14.7 \pm 0.33$ \\
\hline Bpa (4-benzoyl-phenylalanine) & & $5.43 \pm 0.25$ & $15.56 \pm 0.87$ & $28.3 \pm 0.58$ \\
\hline $\mathrm{Nle}(O$-Bzl) (6-benzyloxynorleucine) & & $1.68 \pm 0.11$ & $9.27 \pm 0.35$ & $53.2 \pm 2.55$ \\
\hline Glu(Bzl) (glutamic acid benzyl ester) & & $2.18 \pm 0.05$ & $6.05 \pm 0.26$ & $27.8 \pm 0.18$ \\
\hline
\end{tabular}

Detailed kinetic analysis of fluorogenic substrates of human cathepsin C and DPAP1

In the first step of the analysis, we have focused on the kinetic parameters $\left(K_{\mathrm{m}}, k_{\text {cat }}, k_{\text {cat }} / K_{\mathrm{m}}\right)$ of human cathepsin C for several substrates selected from the P1 and P2 libraries. In the P1 library, we found that the highest enzyme efficiency $\left(k_{\mathrm{cat}} / K_{\mathrm{m}}\right)$ for human cathepsin $\mathrm{C}(5.3 \times$ $\left.10^{6} \mathrm{~s}^{-1} \mathrm{M}^{-1}\right)$ was observed with $\operatorname{Met}-\mathrm{Nle}(O-\mathrm{Bzl})-\mathrm{ACC}$, which is in good agreement with the library screening data. Very good kinetic values were also observed for the other dipeptide substrates with bulky and hydrophobic unnatural amino acids in the P1 position, such as Met-Glu(Bzl), MetBip and Met-Bpa (Table 1).

Analysis of the kinetic parameters for human cathepsin $\mathrm{C}$ in the $\mathrm{P} 2$ position demonstrates a high preference for small aliphatic side chains. The highest enzyme efficiency $\left(k_{\text {cat }} / K_{\mathrm{m}}\right), 1.8 \times 10^{6} \mathrm{~s}^{-1} \mathrm{M}^{-1}$ (human) was found with an unnatural amino acid derivative, Abu (Table 2 ). Other preferred derivatives in the S2 pocket were Hse and Met. All these values are in very good agreement with the library screening data (Fig. 4).

To validate the substrate preferences in the P1 and P2 pockets of human cathepsin $\mathrm{C}$, we designed and synthesized fluorogenic dipeptide substrates that contain the bestrecognized amino acids, $\mathrm{Nle}(O-\mathrm{Bzl})$ in the $\mathrm{P} 1$ position and $\mathrm{Abu}$ in the $\mathrm{P} 2$ position. In parallel, we synthesized and routinely used a commercial substrate for mammalian cathepsin $\mathrm{C}$, which has phenylalanine in the $\mathrm{P} 1$ position and glycine in the $\mathrm{P} 2$ position. Next, we directly compared all of the kinetic parameters of both substrates and found that the substrate we designed with unnatural amino acids 
Table 2 Kinetic parameters $\left(K_{\mathrm{m}}, k_{\mathrm{cat}}, k_{\mathrm{cat}} / K_{\mathrm{m}}\right)$ of selected substrates for human cathepsin $\mathrm{C}$ from the P2 library $\left(\mathrm{NH}_{2}-\mathbf{X}_{\text {-L-hPhe-ACC) }}\right.$

\begin{tabular}{|c|c|c|c|c|}
\hline \multicolumn{2}{|l|}{$\mathrm{NH}_{2}$-X-hPhe-ACC } & \multicolumn{3}{|c|}{ Human cathepsin C } \\
\hline $\mathrm{X}$ : code/name & $\mathrm{X}$ : structure & $K_{\mathrm{m}} \mu \mathrm{M}$ & $k_{\text {cat }} \mathrm{s}^{-1}$ & $k_{\mathrm{cat}} / K_{\mathrm{m}} \times 10^{5} \mathrm{~s}^{-1} \mathrm{M}^{-1}$ \\
\hline Ala (alanine) & $\mathrm{H}_{3}$ & $24.8 \pm 1.78$ & $13.3 \pm 0.88$ & $5.44 \pm 0.29$ \\
\hline Leu (leucine) & $H_{3}$ & $19.6 \pm 1.46$ & $5.00 \pm 0.11$ & $2.44 \pm 0.04$ \\
\hline Met (methionine) & $\mathrm{CH}_{3}$ & $3.6 \pm 0.12$ & $5.59 \pm 0.14$ & $15.2 \pm 0.75$ \\
\hline Nle (norleucine) & $\mathrm{CH}_{3}$ & $9.9 \pm 0.78$ & $8.35 \pm 0.31$ & $8.16 \pm 0.16$ \\
\hline Hse (homoserine) & $\mathrm{OH}$ & $8.8 \pm 0.66$ & $11.4 \pm 0.45$ & $12.5 \pm 0.12$ \\
\hline Abu (homoalanine) & $\mathrm{CH}_{3}$ & $6.3 \pm 0.58$ & $10.4 \pm 0.68$ & $17.7 \pm 0.82$ \\
\hline
\end{tabular}

Each experiment was repeated at least three times

Table 3 Kinetic parameters $\left(K_{\mathrm{m}}, k_{\mathrm{cat}}, k_{\mathrm{cat}} / K_{\mathrm{m}}\right)$ of the best substrate identified and a commercial substrate for human cathepsin C. Each experiment was repeated at least three times

\begin{tabular}{|c|c|c|}
\hline ACC substrate & $\mathrm{NH}_{2}$-Abu-Nle $(O-\mathrm{Bzl})-\mathrm{ACC}$ & $\mathrm{NH}_{2}$-Gly-Phe-ACC \\
\hline Structure & & \\
\hline$K_{\mathrm{m}} \mu \mathrm{M}$ & $1.88 \pm 0.11$ & $167.2 \pm 5.3$ \\
\hline$k_{\text {cat }} \mathrm{s}^{-1}$ & $17.8 \pm 0.56$ & $3.66 \pm 0.11$ \\
\hline$k_{\mathrm{cat}} / K_{\mathrm{m}} \times 10^{5} \mathrm{~s}^{-1} \mathrm{M}^{-1}$ & $94.5 \pm 0.34$ & $0.22 \pm 0.013$ \\
\hline
\end{tabular}

is more than 400 times better than Gly-Phe in terms of $k_{\text {cat }}$ I $K_{\mathrm{m}}$ values. A further analysis of the kinetic parameters demonstrates that the difference between both substrates is primarily seen in the $K_{\mathrm{m}}$ value, which is significantly higher for Gly-Phe (Table 3). Interestingly, the turnover number $\left(k_{\text {cat }}\right)$ did not differ as greatly between these substrates (approximately, five times greater for our substrate than for Gly-Phe).

Finally, we compared the kinetic parameters between human and parasite orthologs with the hope of finding significant differences that would allow us to differentiate the enzymes. Our primary aim was to find a sequence that would be very efficiently recognized by DPAP1, but significantly less so by the human ortholog. Sequence alignment analysis reveals that parasitic DPAP1 shares 24 and $26 \%$ identity with human and bovine orthologs, respectively. This was reflected in significant differences in the library screening data, where the malarial parasite ortholog had substrate specificity distinct from mammalian orthologs. The most striking difference was observed in the P2 position, where the unnatural Pip derivative was recognized very efficiently by DPAP1, but minimally by 
Table 4 Kinetic parameters $\left(K_{\mathrm{m}}, k_{\mathrm{cat}}, k_{\mathrm{cat}} / K_{\mathrm{m}}\right)$ of selected substrates for human and malarial cathepsin C

\begin{tabular}{|c|c|c|c|c|c|c|}
\hline \multirow[t]{2}{*}{ Substrate } & \multicolumn{2}{|l|}{$K_{\mathrm{m}}, \mu \mathrm{M}$} & \multicolumn{2}{|l|}{$k_{\text {cat }}, \mathrm{s}^{-1}$} & \multicolumn{2}{|c|}{$k_{\text {cat }} / K_{\mathrm{m}} \times 10^{-5}, \mathrm{~s}^{-1} \mathrm{M}^{-1}$} \\
\hline & Human & Malarial & Human & Malarial & Human & Malarial \\
\hline Pip-hPhe-ACC & $90.9 \pm 4.91$ & $0.67 \pm 0.14$ & $10.8 \pm 0.16$ & $0.22 \pm 0.004$ & $1.16 \pm 0.11$ & $3.22 \pm 0.53$ \\
\hline Pip-Lys-ACC & $77.4 \pm 5.35$ & $0.18 \pm 0.01$ & $7.45 \pm 0.41$ & $0.12 \pm 0.0004$ & $0.96 \pm 0.07$ & $6.59 \pm 0.37$ \\
\hline
\end{tabular}

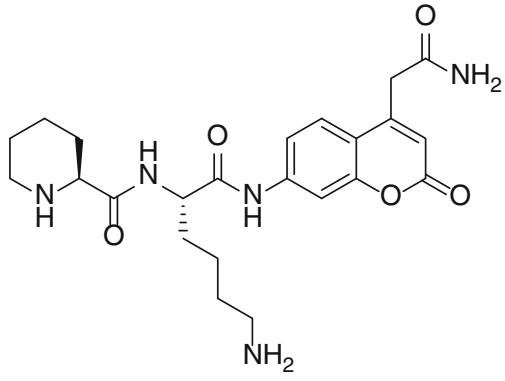

Fig. 5 Structure of the optimal substrate for malarial DPAP1

mammalian orthologs. The comparison of kinetic parameters for the P2 library sequence Pip-hPhe confirms the library-based data. DPAP1 hydrolyzed this sequence three times more efficiently $\left(k_{\text {cat }} / K_{\mathrm{m}}-3.22 \times 10^{5} \mathrm{~s}^{-1} \mathrm{M}^{-1}\right)$ than the human ortholog $\left(k_{\mathrm{cat}} / K_{\mathrm{m}}-1.16 \times 10^{5} \mathrm{~s}^{-1} \mathrm{M}^{-1}\right)$ (Table 4). To further improve the efficiency of the substrates, we designed a DPAP1 substrate, Pip-Lys, with an optimal sequence in the $\mathrm{P} 2$ positions and Lys in $\mathrm{P} 1$ position (Fig. 5). Since Nle( $O$-Bzl) is the most preferred by all three tested proteases in P1, in search for specific DPAP1 sequence we decided to use Lys, which is not predominantly preferred by human orthologs and is second best for DPAP1. Kinetic analysis reveals that this substrate is approximately two times better in terms of its $k_{\mathrm{cat}} / K_{\mathrm{m}}$ value $\left(6.59 \times 10^{5} \mathrm{~s}^{-1} \mathrm{M}^{-1}\right)$ than the sequence with hPhe in the P1 position $\left(3.22 \times 10^{5} \mathrm{~s}^{-1} \mathrm{M}^{-1}\right)$ (Table 4). Pip-LysACC is also more specific toward DPAP1. It is more than six times more active $\left(k_{\mathrm{cat}} / K_{\mathrm{m}}\right)$ than human cathepsin $\mathrm{C}$ (Table 4).

\section{Discussion}

Due to the implication of human cathepsin C in PapillonLefevre disease and Haim-Munk syndrome or inflammatory diseases, and the involvement of its malarial ortholog DPAP1 in the hemoglobin digestion pathway, both enzymes are considered interesting medical targets (Deu et al. 2010; Guay et al. 2010; Klemba et al. 2004). It is especially intriguing that during parasite infection, both enzymes are found in the human (DPAP1 is found in human red blood cells infected with $P$. falciparum parasite, while human cathepsin $\mathrm{C}$ is a lysosomal protease), and therefore molecules designed to specifically reach the DPAP1 active site must significantly differentiate between human and parasite orthologs. Due to the involvement of human cathepsin $\mathrm{C}$ in activation of several serine proteases in inflammatory or immune cells, cross-reactivity of potential inhibitor of DPAP1 with human ortholog might result in detrimental side effects. Studies published to date regarding the differentiation between human cathepsin $\mathrm{C}$ and DPAP1 have only been partially successful; new tools are required to solve this problem (Wang et al. 2011).

In our studies, we have applied a new approach for the selection of very active and selective substrates for these aminodipeptidases. First, we have designed and synthesized a library of individual fluorogenic dipeptide substrates to probe the S1 and S2 pockets of the enzymes. The major advantage of this library is its composition, which includes both natural and unnatural amino acids. Unnatural amino acids were selected from different structural groups of compounds (aliphatic, aromatic, bulky hydrophobic, D-amino acids, cyclic) to cover all possible interactions in the binding pockets. This library allowed us to directly compare three orthologs of cathepsin $\mathrm{C}$, human, bovine and malarial parasite. We found that the human and bovine orthologs have almost identical substrate specificity in their $\mathrm{S} 1$ and S2 pockets. They prefer bulky, aromatic residues in the $\mathrm{P} 1$ position and rather small and aliphatic amino acids in the $\mathrm{P} 2$ position. These data are in good agreement with previously published reports, in which some selected unnatural amino acids were used. For example, Tran et al. found that P1-position homophenylalanine in the fluorogenic substrate Gly-hPhe-AMC is more efficiently hydrolyzed by bovine cathepsin $\mathrm{C}$ than its one-methylene-groupshorter natural homolog, phenylalanine (Gly-Phe-AMC) (Tran et al. 2002). In the same work, the authors demonstrated that bovine cathepsin $\mathrm{C}$ barely recognizes Gly at the $\mathrm{P} 2$ position, but quite efficiently recognizes Ala, Abu, Nva and Nle. These data are also in good agreement with our library screening results. In another approach, $\mathrm{Li}$ et al. (2009) designed efficient rhodamine-based substrates for human cathepsin $\mathrm{C}$ with hPhe or Bip in the $\mathrm{P} 1$ position and $\mathrm{Abu}$ in the P2 position. The overall preference for quite 
broad substrate specificity in the $\mathrm{P} 1$ position of mammalian orthologs and rather narrow substrate specificity in the P2 position can be explained by the analysis of available crystal structures (Turk et al. 2001; Dahl et al. 2001; Molgaard et al. 2007). The S1 pocket is located on the surface of the enzyme and is exposed to the solvent. Its large size demonstrates that it preferentially accommodates very bulky and hydrophobic residues, such as these found in our studies. We assume that the large size of the residues results in more interactions with the surface of the enzyme and thus increases its affinity to the substrate. This hypothesis can by confirmed by the kinetic data we obtained for $\mathrm{NH}_{2}$-Abu-Nle $(O-\mathrm{Bzl})-\mathrm{ACC}$ and $\mathrm{NH}_{2}$-GlyPhe-ACC, which differ very significantly in terms of the $k_{\text {cat }} / K_{\mathrm{m}}$ value, but not as significantly in the $k_{\text {cat }}$ value (Table 3). The determining factor here is the $K_{\mathrm{m}}$ value, which reflects, to a significant extent, the binding affinity of the substrate. Further analysis of the human cathepsin $\mathrm{C}$ crystal structure demonstrates that the $\mathrm{S} 2$ pocket is rather long and narrow. This explains why rather small and aliphatic amino acids, such as Ala, Abu, Met, Hse, Nle or Nva, are preferred in the $\mathrm{P} 2$ position. The structure of the optimal substrate $\mathrm{NH}_{2}$-Abu-Nle $(O$-Bzl $)$-ACC can also be used in further studies for the design of inhibitors or activity-based probes for cathepsin C. The observed substrate specificity also demonstrates that bovine cathepsin $\mathrm{C}$, which is much more readily available and less expensive, can substitute for the human ortholog in routine studies on new chemical tools.

In the studies with malarial DPAP1, we provide new evidence that this enzyme quite significantly differs in substrate specificity from mammalian orthologs. In contrast to human and bovine cathepsin C, DPAP1 demonstrates very little preference in the P1 position toward bulky and hydrophobic amino acids of phenylalanine like structure and efficiently hydrolyzes amino acids with long aliphatic side chains with aromatic group at the end (Nle $(O-\mathrm{Bzl})$, Glu(Bzl)) or with basic side chains, Arg and Lys. As a result of library screening studies, we have found that DPAP1 preferentially cleaves the unnatural cyclic amino acid Pip in the P2 position, which is barely tolerated by human orthologs. These results allowed us to design an optimal DPAP1 substrate, Pip-Lys-ACC, for which the $k_{\text {cat }} /$ $K_{\mathrm{m}}$ values were calculated. With $k_{\text {cat }} / K_{\mathrm{m}}$ values equal to $6.59 \times 10^{5} \mathrm{~s}^{-1} \mathrm{M}^{-1}\left(\right.$ DPAP1) and $0.96 \times 10^{5} \mathrm{~s}^{-1} \mathrm{M}^{-1}$ (cathepsin $\mathrm{C}$ ), we have demonstrated that this substrate is quite selective toward the malarial ortholog. On the other hand, this might seem quite low given the huge difference in the initial screen. The reason is that DPAP1 is 30-100 times less efficient than cathepsin $\mathrm{C}$ using natural amino acid dipeptide substrates, as demonstrated by Wang et al. (2011). It is then apparent that the specificity has been shifted $\sim 100$-fold in favor of DPAP1.
The analyses presented here focused on the S1 and S2 pockets, but it is also likely that enhanced interactions with natural peptide and protein substrates utilize interactions at the C-terminal side ( $\mathrm{S}^{\prime}$ side) of the scissile bond. Unfortunately, there is no currently available technology to probe the $S^{\prime}$ site of aminopeptidases with synthetic substrates.

In conclusion, we have designed and tested for the first time a tailored fluorogenic substrate library containing natural and unnatural amino acids to define the specificity of the active site of human, bovine and malarial cathepsin C. Our results clearly demonstrate very significant differences in the preference for binding of both natural and unnatural amino acids to the $\mathrm{S} 1$ and $\mathrm{S} 2$ pockets between mammalian and malarial orthologs. The catalytic rates of hydrolysis for substrates with unnatural amino acids designed based on library screening were significantly improved, proving the utility of this approach. For example, for human cathepsin C, we have obtained a substrate that is more than 400 times better in terms of $k_{\mathrm{cat}} / K_{\mathrm{m}}$ than the commonly used commercial substrate. The observed significant differences in terms of the structure of the substrates between human and malarial orthologs can be used for the design of specific inhibitors or activity-based probes (ABPs). Finally, the methodology used here provides the proof of concept for the application of this library in screening other types of diaminopeptidases or for the direct comparison of enzyme orthologs from different organisms.

Acknowledgments This work was supported by grant number NN 302 276437. Marcin Drag and Marcin Poreba are grateful to the Foundation for Polish Science for support. The research was supported by Wroclaw Research Center EIT+ under the project Biotechnologies and Advanced Medical Technologies ("BioMed") (POIG 01.01.02-02-003/08-00) financed by the European Regional Development Fund (Operational Programme Innovative Economy, 1.1.2).

Conflict of interest The authors declare that they have no conflict of interest.

Open Access This article is distributed under the terms of the Creative Commons Attribution License which permits any use, distribution, and reproduction in any medium, provided the original author(s) and the source are credited.

\section{References}

Adkison AM, Raptis SZ, Kelley DG, Pham CT (2002) Dipeptidyl peptidase I activates neutrophil-derived serine proteases and regulates the development of acute experimental arthritis. J Clin Invest 109(3):363-371. doi:10.1172/JCI13462

Arastu-Kapur S, Ponder EL, Fonovic UP, Yeoh S, Yuan F, Fonovic M, Grainger M, Phillips CI, Powers JC, Bogyo M (2008) Identification of proteases that regulate erythrocyte rupture by the malaria parasite Plasmodium falciparum. Nat Chem Biol 4(3):203-213. doi:10.1038/nchembio.70 
Dahl SW, Halkier T, Lauritzen C, Dolenc I, Pedersen J, Turk V, Turk B (2001) Human recombinant pro-dipeptidyl peptidase I (cathepsin C) can be activated by cathepsins $\mathrm{L}$ and $\mathrm{S}$ but not by autocatalytic processing. Biochemistry 40(6):1671-1678 pii: bi001693z

Deu E, Leyva MJ, Albrow VE, Rice MJ, Ellman JA, Bogyo M (2010) Functional studies of Plasmodium falciparum dipeptidyl aminopeptidase I using small molecule inhibitors and active site probes. Chem Biol 17(8):808-819. doi:10.1016/j.chembiol.2010. 06.007

Gocheva V, Joyce JA (2007) Cysteine cathepsins and the cutting edge of cancer invasion. Cell Cycle 6(1):60-64

Guay D, Beaulieu C, Percival MD (2010) Therapeutic utility and medicinal chemistry of cathepsin $\mathrm{C}$ inhibitors. Curr Top Med Chem 10(7):708-716

Hart TC, Hart PS, Bowden DW, Michalec MD, Callison SA, Walker SJ, Zhang Y, Firatli E (1999) Mutations of the cathepsin C gene are responsible for Papillon-Lefevre syndrome. J Med Genet 36(12):881-887

Hart TC, Hart PS, Michalec MD, Zhang Y, Firatli E, Van Dyke TE, Stabholz A, Zlotogorski A, Shapira L, Soskolne WA (2000) Haim-Munk syndrome and Papillon-Lefevre syndrome are allelic mutations in cathepsin C. J Med Genet 37(2):88-94

Ken McDonald J, Callahan PX, Ellis S (1972) [22] Preparation and specificity of dipeptidyl aminopeptidase I. Methods Enzymol 25C:272-281. doi:10.1016/S0076-6879(72)25024-8

Klemba M, Gluzman I, Goldberg DE (2004) A Plasmodium falciparum dipeptidyl aminopeptidase I participates in vacuolar hemoglobin degradation. J Biol Chem 279(41):43000-43007. doi:10.1074/jbc.M408123200

Krugliak M, Zhang J, Ginsburg H (2002) Intraerythrocytic Plasmodium falciparum utilizes only a fraction of the amino acids derived from the digestion of host cell cytosol for the biosynthesis of its proteins. Mol Biochem Parasitol 119(2):249-256

Kudo M, Melton AC, Chen C, Engler MB, Huang KE, Ren X, Wang Y, Bernstein X, Li JT, Atabai K, Huang X, Sheppard D (2012) IL-17A produced by alphabeta $\mathrm{T}$ cells drives airway hyperresponsiveness in mice and enhances mouse and human airway smooth muscle contraction. Nat Med 18(4):547-554. doi:10. 1038/nm.2684

Li J, Petrassi HM, Tumanut C, Masick BT, Trussell C, Harris JL (2009) Substrate optimization for monitoring cathepsin C activity in live cells. Bioorg Med Chem 17(3):1064-1070. doi:10.1016/j.bmc.2008.02.002

Maly DJ, Leonetti F, Backes BJ, Dauber DS, Harris JL, Craik CS, Ellman JA (2002) Expedient solid-phase synthesis of fluorogenic protease substrates using the 7-amino-4-carbamoylmethylcoumarin (ACC) fluorophore. J Org Chem 67(3):910-915 jo016140o

McGuire MJ, Lipsky PE, Thiele DL (1993) Generation of active myeloid and lymphoid granule serine proteases requires processing by the granule thiol protease dipeptidyl peptidase I. J Biol Chem 268(4):2458-2467

Molgaard A, Arnau J, Lauritzen C, Larsen S, Petersen G, Pedersen J (2007) The crystal structure of human dipeptidyl peptidase I (cathepsin C) in complex with the inhibitor Gly-Phe-CHN2. Biochem J 401(3):645-650. doi:10.1042/BJ20061389

Pham CT, Ley TJ (1999) Dipeptidyl peptidase I is required for the processing and activation of granzymes A and B in vivo. Proc Natl Acad Sci USA 96(15):8627-8632

Ragheb D, Dalal S, Bompiani KM, Ray WK, Klemba M (2011) Distribution and biochemical properties of an M1-family aminopeptidase in Plasmodium falciparum indicate a role in vacuolar hemoglobin catabolism. J Biol Chem 286(31):27255-27265. doi:10.1074/jbc.M111.225318

Tallan HH, Jones ME, Fruton JS (1952) On the proteolytic enzymes of animal tissues. X. Beef spleen cathepsin C. J Biol Chem 194(2):793-805

Tanaka TQ, Deu E, Molina-Cruz A, Ashburne MJ, Ali O, Suri A, Kortagere S, Bogyo M, Williamson KC (2013) Plasmodium dipeptidyl aminopeptidases as malaria transmission-blocking drug targets. Antimicrob Agents Chemother 57(10):4645-4652. doi:10.1128/AAC.02495-12

Tran TV, Ellis KA, Kam CM, Hudig D, Powers JC (2002) Dipeptidyl peptidase I: importance of progranzyme activation sequences, other dipeptide sequences, and the N-terminal amino group of synthetic substrates for enzyme activity. Arch Biochem Biophys 403(2): $160-170$

Turk D, Janjic V, Stern I, Podobnik M, Lamba D, Dahl SW, Lauritzen C, Pedersen J, Turk V, Turk B (2001) Structure of human dipeptidyl peptidase I (cathepsin C): exclusion domain added to an endopeptidase framework creates the machine for activation of granular serine proteases. EMBO J 20(23):6570-6582. doi:10. 1093/emboj/20.23.6570

Wang F, Krai P, Deu E, Bibb B, Lauritzen C, Pedersen J, Bogyo M, Klemba M (2011) Biochemical characterization of Plasmodium falciparum dipeptidyl aminopeptidase 1. Mol Biochem Parasitol 175(1):10-20. doi:10.1016/j.molbiopara.2010.08.004

Yuan F, Verhelst SH, Blum G, Coussens LM, Bogyo M (2006) A selective activity-based probe for the papain family cysteine protease dipeptidyl peptidase I/cathepsin C. J Am Chem Soc 128(17):5616-5617. doi:10.1021/ja060835v 